\title{
HR intranets in France: A Longitudinal Study
}

\author{
Veronique Guilloux ${ }^{1}$, Michel Kalika ${ }^{2}$ and Florence Laval ${ }^{3}$ \\ ${ }^{1}$ Faculté Sciences Humaines, LEA IRG, 61, Avenue du Général de Gaulle \\ 94010 Créteil, France \\ ${ }^{2}$ Université Paris Dauphine \\ CREPA, Place du Maréchal de Lattre de Tassigny \\ 75775 Paris Cedex 16, France
}

${ }^{3}$ IAE, Cerege, 20, rue Guillaume VII le Troubadour BP 63986022 Poitiers, France

\begin{abstract}
The article presents some interesting results of an exploratory and longitudinal study (1998-2004) based on a sample of French firms. The context of the Introduction of intranets, the development stages, performance and intranets contents are presented. Three approaches exist: corporate intranet, specialised intranet and HR intranet. They can be linked to different stages of development more or less sophisticated: communication, functional support, knowledge management. The HR Function will be a winner if it integrates the intranet in its management process and if the actors are aware of the strikes and if they behave as real change agents.
\end{abstract}

\section{Introduction}

The article presents some interesting results of an exploratory and longitudinal study based on a sample of French firms. The survey identifies the diffusion of HRM practices, as well as their development stages. The investigation aims at answering the following questions: How intranet networks go with the HR competence development? How could they support the HR Function? What are the best French practices linking ITC and skills management?

To confer on the «Intranet d'or », the CEGOS proceeds a ranking of firms based on their innovating Intranet functionalities, grounded on their utility, their techniques and their ergonomics. The number of analysed dossier is in 1998 of 19, in 199919 , 2000, 21, 2001 19, 2002 33, 2003, 12 and 200427 and has led to numerous articles [1], [2], [3], [4]. The best innovating uses in skills development involve: E-Learning applications, Mobility applications, Expertise management, Capitalization, Knowledge transfer and professional practices.

The questionnaire is submitted as an application file included open questions on the firms and its intranet (techniques, cost, assessment and perspectives). Few empirical studies concerning intranet exist. That's why Cegos concrete data show some interest The article here analyses the stakes and the organisational and strategical risks linked with the use of HR intranet for the HR function. Theoretical IT concepts and techno- 
logical infusion aspects are analysed as the results go by. The context of the Introduction of intranets, the development stages, performance and intranets contents are presented.

\section{The Different Contexts of the Introduction of HR Intranets}

The available data issued from the exploratory study concerning intranet used as HRM support could be gathered into three categories: the context variables ( environment, strategy, HRM); the variables characterizing the intranets (objectives, functionalities, technique, budget, implementation date, number of workers concerned); the results (encountered difficulties, immediate results, appraisals and perspectives). For each variable, the data aggregation permits to draw significant axes. A managerial case study will illustrate examples of intranet functions. Beforehand the next paragraph describes the sample and the context of HR intranet introduction

\subsection{Sample: Firms Size And Activity Sectors}

Even if the medium size of the companies using an intranet is high, intranets could be introduced in medium firms or even in SME. In our sample $26 \%$ of the firms have less than 1000 employees; $28 \%$ belong to the category (1000-4999 employees); $32 \%$ are a member of the group (5000-49999) and 14\% have more than 50000 employees. The number of employees is not a distinguishing factor for an intranet ownership. The employees' geographical dispersal and the intranet accessibility are not a privilege for big companies. The professionals who have answered, are issued from the public sector (19\% hospital, town council, chamber of commerce) as well as private sectors (service activity (banks, insurances) $28 \%$, industries $35 \%$; ITC (telecommunication, computing, software conception) : $18 \%$.

\subsection{Context Variables Of The Intranets: Environment, Strategy And HRM}

Environment: Environment is a contingent factor for the ITC and intranet introduction in the organisations. In the present sample, around $18 \%$ of the firms are confronted to an intensive information environment. The public environment is not really competitive (example EDF) although most of them are now internationally acting.

Organisation and Strategy. The staff is geographically scattered in subsidiaries, establishments, production sites, agencies. It can also be individual employees (« nomad» workers, agents, experts). Most of the time, the group which is linked to our sample is multinational. It is represented with divisional, matrix or reticular organizational configurations. Structurational criterium is geographical. These organisations are global, international, transnational, or spread out through the French territory. Organisational integration takes on a strategic aspect. Subsidiaries, unities, work groups and individuals are also concerned. Virtual teams resorted to ITC are com- 
monly held. The literature on that theme is from now on copious. In most of the firms (86\%), the intranet development corresponds to an integrational strategy. It comes up with internal networks development or corresponds to a post-fusion situation and in general it reflects a will to accelerate the integration of historically geographically spread out unities. The existence of an HR function is also a permanent feature in the firms, but the integration level of the intranet by the HRF and its impact on HRM are very diverse. The strategic HRM context of the intranet setting up could be also pertinent, notably the tool functionalities and its performance. The dossiers which have been analyzed reveal the existence of two significant contexts: $-23 \%$ of the firms are reorganizing themselves or reengineering one or more processes. During the implementation of intranet, firms are changing their management processes. $-86 \%$ of the companies are underlining the necessity of organizational integration either because they are organized in network or because they have carried out external growth operations (fusions, acquisitions) or internal expansions. They want to create or reinforce the cohesion, an homogeneous culture, the activities coordination and cross processes. The intranet comes in support to interorganisational relationships.

Human Resource Management. A US typology concerning the link between HRM and performance demonstrates that $43 \%$ of the groups run a traditional hr policy». It means that the classical functions are realized (employment, compensation, training and participation) as well as certain projects such as flexible working hours (time budgeting, reduced working week). This strategy aims at bettering the employees' mobilisation and the working performance. In our study, a means of $45 \%$ of the firms are kept in a such strategic or innovating HR policy. The function experts conceive competency management and carrier management systems besides usually functions. Organizational knowledge is also developed when the group qualifies itself as "a learning company" or develops «management learning » policy. Furthermore, $12 \%$ of the firms have a function in evolution, either because the enterprise is young or because of a reorganisation. In that case, the goal is to rationalize and redeploy its HR policies. They invest for example, on that occasion in a new HRM-IS.

\section{Development Stages and HR Intranet}

The article will offer an overview of applications and detailed Intranet illustrations. The aggregation of information issued from approximately 150 files reveals the targets of the firms and the level of the intranet sophistication. The results, the performance bellow expectation as well as the perspectives of the firms are registered.

The firms in the present sample have adopted one of the three approaches: Specific HR headings can be introduced in a Corporate intranet. The latter is conceived by CEO with the help of the communication and IS department. It's dedicated to the internal organizational communication and to the employees' information. This kind of intranet is at the boundary of HR questionings. The HR intranet, driven by the HR department is a support to the HR policy and to social management practices. They are specific to the HR function and they could be integrated to the corporate intranet. This sort of Intranet integrates functionalities such as access to training cata- 
logues, mobility management, employees administrative management. Finally, specialized intranets are developed for training, sharing knowledge and competence development problematic require. They are implemented by the HR department or by $\mathrm{CEO}$, when the stakes are highly strategic or dedicated to a specific goal.

Among the available dossiers, $32 \%$ concerns a specialised HR intranet, $31 \%$ a HR generalist intranet, and $37 \%$ a corporate intranet. As time goes by, corporate intranets grow.The corporate intranet is now very common and it can be associated with the notion of corporate intranet Portal. An enterprise portal is the one entry point to the intranets and all of its content and applications.

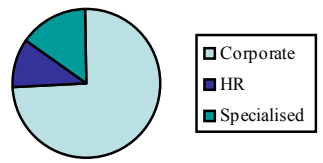

Fig. 1. 1998-2001.

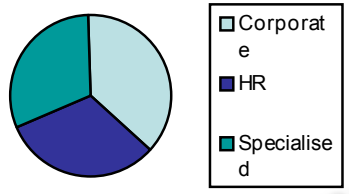

Fig. 1b. 2004.

On the average, specialized Intranet is more expensive. This has been verified for several years. Specialized intranets developing E-learning, KM imposes specific developments while the other categories only imply online information.

The intranet differences in cost are coherent with the time limit realization which was of 25 months in 1998, of 10-15 months in 2000 of 8 months in 2002. This trend is the same whatever the type of intranet. It reveals scales economies.

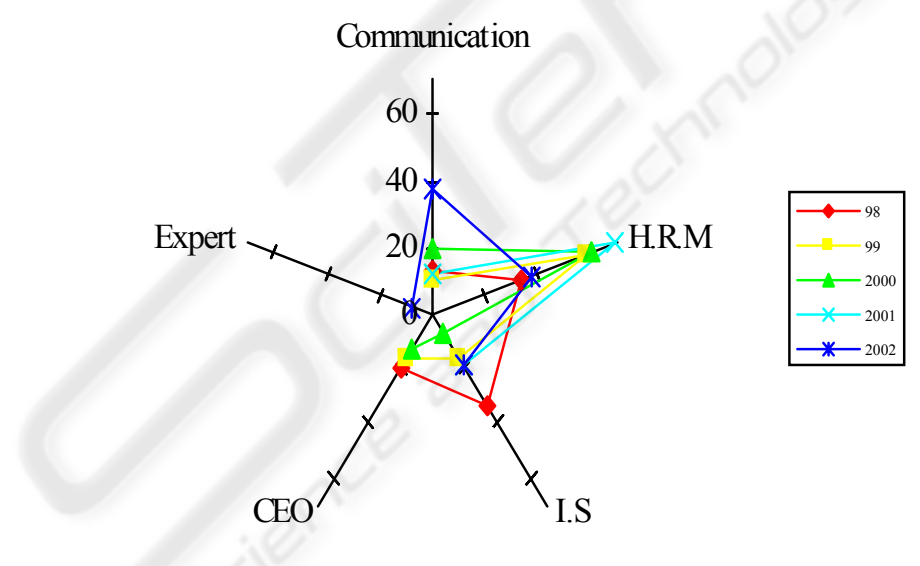

Fig. 2. Project supervision.

The supervision of an intranet project is done for more than $50 \%$ by the HR department. This percentage is not surprising. However the fact that in $50 \%$ of the case, the HR department does not run the project is odd! The diagram clearly tells the contribution of the other functions (IS, Communication, experts).

The profile of the project manager depends on the nature of the intranet. $77 \%$ of the projects are attributed to HR managers. For the corporate Intranet, the managing of the HR department is less frequent and is usually assumed by the communication director or the CEO. As far as specialized Intranets managing is concerned, the IS 
department is responsible. The technical specificity of certain functionalities is justified by such a fact.

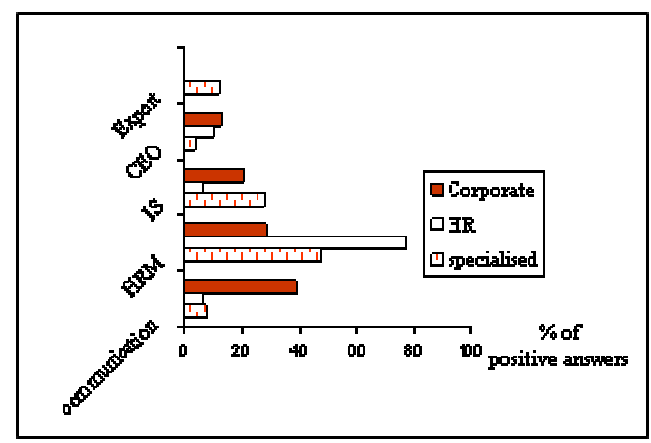

Fig. 3. Project supervision/ intranet type.

There is an evolution in the project management coordination. In 1998 the project supervisor has more important than the project owner. In 2004, the opposite is noticed: the functional department has the leadership role.

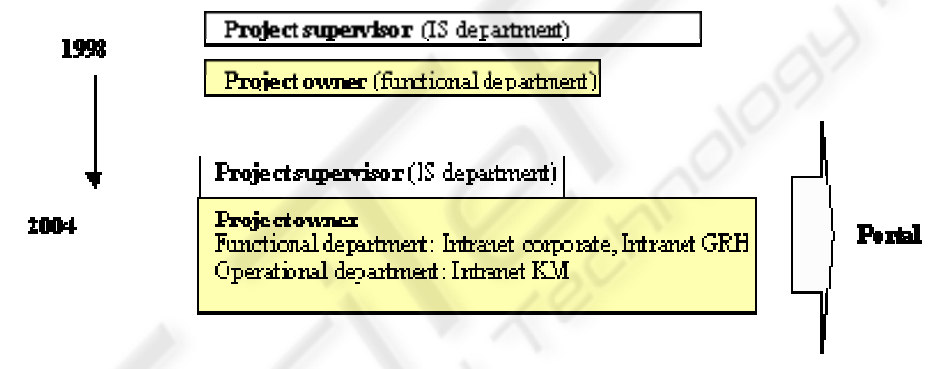

Fig. 4. Project supervisor / Project owner.

\section{The Functionalities of the HR Intranet}

The analysis of the content of the intranets shows the diversity of the proposed services and a large diversity of the intranet configurations. Some of them cover all the functionalities listed bellow and reveal the generalist side of the applications; others are focused on a sole function.

The analysis of the observations issued from our sample offer a precise idea of the content used in an Intranet supporting HR policy:

-The HR information headings often contain a description of the firm HR procedures, legal items (employment law collective agreement, company-wide agreement), works council reports, welfare benefits, mission statement, and directories.

-The mobility and carrier headings diffuse job offers, job description, frame of reference for each occupation, personal transfers, and procedures for the job application. 
-The training policy is enhanced by a learning catalogue, training programme, formalities, online admission forms. E learning modules could be at the disposal of users.

-The knowledge capitalisation relies on databases, groupware, forums, suggestion box.

-An electronic administrative management permits to realise online tasks (annual leave, expense account, time and attendance management, updating of the employees' file ...)

-The competence management is mediated by the diffusion of the competences frame of reference, the modalities of acquisition of knowledge and the assessment.

A configuration of an intranet conceived as a support of HR, combines headings that informs the employees and that automates certain management practices via ITC.

The analysis of the functionalities by Intranet types underlines several differences. Specialised intranets are particularly concerned by knowledge capitalisation and competence management. HR intranet and corporate intranet promote HR information, mobility and career items.

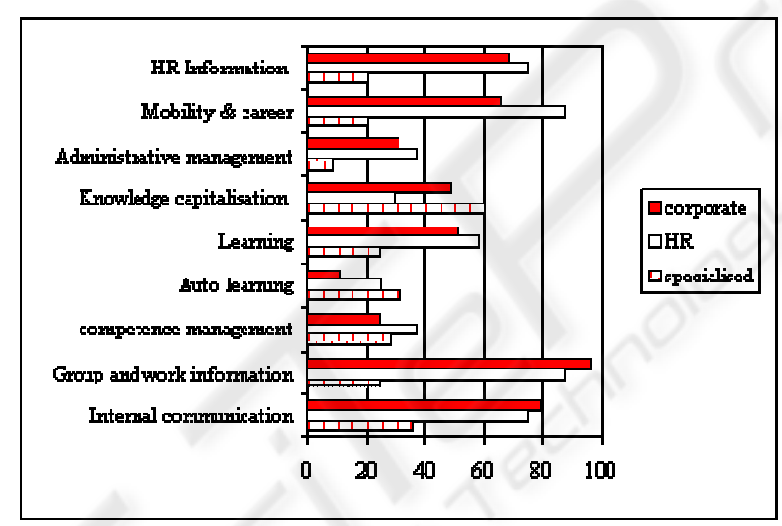

Fig. 5. Intranet Functionalities.

During the years of observation, mobility and careers, administrative management and competence management are approved.

\section{Performance of the HR Intranet}

The article lists the difficulties faced by the managers. They analyse the difficulties tied with the intranet working and the different point of view of the final user. Their model is based on the Technological Acceptance Model and on several items Users and CEO's involvements, Accessibility and use easiness, Perceived utility.

\subsection{Difficulties}

The difficulties identified in the French firms are various: 
-Organisational and cultural problems: the absence of Intranet culture and the resistance to change management, resistance to transparence \& transversality, are common. Indeed, the Intranet destructures and hustles the usual information circuits.

-Problems of use: the risk is that the Intranet is perceived as a gadget, or something, lacking of user-friendliness; A less used forum, an absence of real division of knowledge are a barrier to the use of the tools. Moreover, it is difficult to interest heterogeneous populations if one does not create fields of access per type of population.

-Technical problems at the various stages of the project: for example upstream, validation of the graphic charter, then filling of the data base, conversion of the document on HTML page; thereafter, the problems of operation of the navigation software, reliability of the server, heterogeneity of the data-processing park (unloading time, quality of the images) can occur.

-Actualization and integration problems: The absence of a webmaster is a handicap. Indeed, it's necessary to have up to date information in real time and to control the coherence and the validity of information. The head of project will have sometimes to articulate or to integrate several versions of Intranets.

-Safety and confidentiality problem: it is for example said, that the facility of consultations of the results of a $360^{\circ}$ evaluation can encourage not to go further. Companies had to face the hacking of the PC put in free access. The risk of escape of knowledge is thus real.

-Problem of groupware: daring asking questions, learning how to work in team, formalizing the constitution of virtual teams, making collaborate the IS and communication departments, implying the information system department are major challenges. -Problem of non integration of the end-user in the design: an example concerns the weak frequentation of the forums before the employees determine by themselves the results of the subjects of discussion. The advantage and the coherence of the tool are not demonstrated and the user's needs are badly encircled.

-Problem of the financing: the development of the tools, the network and the deployment for the employees (in particular those who do not have a data-processing station and to who it must be necessary to justify the access progressiveness). All this requires a budget and an evaluation of the added value of the Intranet.

Specialized intranets are more concerned by technical difficulties. HR intranets are tied with organizational and updating difficulties. Corporate intranets are confronted by ergonomic difficulties, inter-functional coordination and the non implication of final users. More than $50 \%$ of the firm mentions difficulties of organisational nature before the fact of having technical problems. The latter are tied with the applications implementation, the data updating and the ergonomics of the system. Certain enterprises have mentioned as well the inter-organisational coordination.

\subsection{Key Success Factors}

Regarding the experience of the firms implementing an intranet, key success factor are listed. 


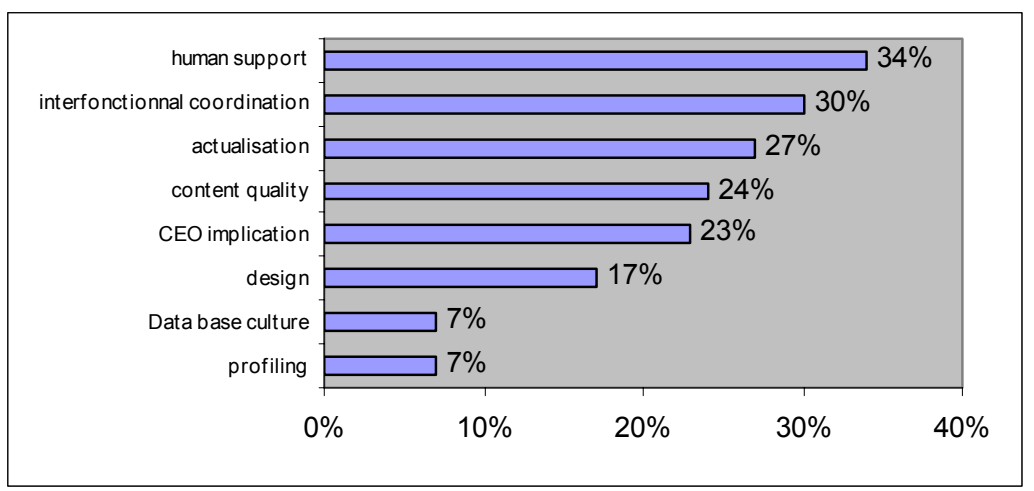

Fig. 6. Success factors.

The human support is a real key success factor in the implementation of the intranet. This human facet is mentioned in one third of the contracts. Problematic of interfunctional coordination follows. The transversal side of intranet projects, the necessary implication of the HR department as well as those of IS and communication services make the project complex.

The key factor for an HR intranet is the Human support; for the Corporate intranet interfunctional coordination appears to be important and for the specialised intranet customisation is quoted (example profiling).

From 1998 to 2001, priorities are more or less the same. In 1998, interfunctional coordination is above all evoked. In 1999, the items concerning human support are chosen. In 2000, the CEO's implication and the quality of the content have priority over the others. In 2001, human support and interfunctional coordination are approved.

\subsection{Results Issued by the Intranet Use}

The items used to evaluate an intranet can be classified by type of intranet technology used. The idea of the tool performance (observed or perceived) is intrinsic, organisational and economic. In fact the assessment can concern technical and social dimensions of the technology but these immediate effects contribute in the long term to the improvement of the organizational collective functioning. Organisational impacts are noticed in terms of coordination, of integration, and culture. Employee's working performances are affected for the same reason as those of management systems. In the present case, the article shows that the analysis must focus on the analysis of IS and on the HRIS. The managing actors define the tool value creation or the Return on investment from productivity indicators and the quality of services.

Items of performance are chosen by the firms and can be presented in three categories:

-Economic performance: is linked with the theme of quality and productivity: improving of the quality, reduce of the cost.

-Performance of the intranet in terms of social or technical facets 
-Organisational performance: culture (diffusion and promotion of the organisational culture, development of an IT culture), integration, coordination, control (federating role towards the divisions, emulation between divisions, facility and efficacy of the sharing of the various resources, network working, transversal communication), internal and external coherence of the HRM system (improving of the internal image of the HR function, reengineering of the working methods of the HR function, automation of the administrative working, formalisation and rationalisation of the HR practices), working performance (new methods of working; e-learning, groupware).

The intrinsic performance of an intranet (technical facet as the number of connexions and social facet and the users satisfaction) is more mentioned than the extrinsic performance (economical, organisational). In 1998, organizational performances are mentioned because there is a lot of corporate Intranets. In 1999, 2000, 2001 professionals evoke some intrinsic performances. Specialised and HR intranets are more oriented toward an intrinsic performance. Implementation of corporate intranets is to the advantage of extrinsic performance because they support communication and are tied with collective stakes.

Intranet applications perspectives can be gathered in two axes: technical development and integration. These empirical elements can be integrated in existing theoretical frames.

Technical development concerns : function of existing items; new items creation; Generalization of the access (to all the divisions or working unities, all the operational positions), facilitation of the implementation of online information in order to enrich the intranet by the majority of the employees. Facilitation of the online implementation

Technological integration aims at decompartmentalizing the specialised intranet and at decompartmentalizing the HR intranet and tied it to other intranets, to the company portal and to the firm ERP. This is the concept of "web centric enterprise " and of the articulation of internet-intranet-extranet.

\section{Conclusion}

The exploratory study on intranets used as an HR support has permit to identify some context variables, some variables characterising intranets and resulted variables. The managerial interest lies in the promotion of potential applications and their accessibility.

Concerning the variables of the context, if the number of employee is not a locking factor, the environment strongly stimulates the intranet introduction in the firm. The strategies of the firm are various: reorganisation, followed by a reengineering of their management processes; research of «excellence» innovation and quality, improving productivity or organisational integration. These conditions justify the technological infusion and more particularly HR support activities. Regarding the HR function, the firm development is significant with an innovative HR management or a traditional HR management or a reorganization of the function. In the first case the HR function could be the « designer » of the intranet. In the second case, experts of the HR func- 
tion are "users" of the intranet. If a big restructurating is engaged, we could think of a stronger implication of the HR department in the intranet development.

Concerning the variables describing the intranets: aims, development level, functionalities are listed. Three approaches exist: corporate intranet, specialised intranet and HR intranet. They can be linked to different stages of development more or less sophisticated: communication, functional support, knowledge management. The various functionalities are: HR information, mobility and careers, administrative management, knowledge capitalisation, training, competence management. Firms integrate these items and develop them according the aim assigned to the intranet and according to the degree of strategic integration of the HR function.

The difficulties are various and tied with organisational issues, technical, updating, security, confidentiality, finance reasons etc ... Problems can emerge as well when the final user is not involved in the design stage.

Intranet performances are evaluated in economic, technical, social and organisational terms. The HR Function will be a winner if it integrates the intranet in its management process and if the actors are aware of the strikes and if they behave as real change agents.

\section{References}

1. Laval F., Guilloux V., Kalika M. (2005), L'intranet RH : de l'E-RH au Knowledge Management in Michel Kalika, Véronique Guilloux, Florence Laval, et Mohammed Matmati, ERH réalités manageriales, ed Vuibert.

2. Guilloux V., Laval F., Kalika M. (2005) Les intranets RH : de l'introduction des TIC aux nouvelles formes d'organisation in Michel Kalika, Véronique Guilloux, Florence Laval, et Mohammed Matmati, E-RH réalités manageriales, ed Vuibert.

3. Laval F., Guilloux V., Kalika M. (2002), Les intranets RH: pratiques des entreprises et problématiques, dans M. Kalika, E-GRH: Révolution ou évolution?, Editions Liaisons.

4. Kalika M., Laval F. (2003), E-management et ressources humaines, Encyclopédie des Ressources Humaines, Economica. 